\title{
RADIOMETRIC CALIBRATION OF MARS HIRISE HIGH RESOLUTION IMAGERY BASED ON FPGA
}

\author{
Yifan Hou ${ }^{\text {a, b, *, Xun Geng }}{ }^{\text {a }}$, Shuai Xing ${ }^{\text {a }}$, Yonghe Tang ${ }^{\text {b }}$, Qing Xu ${ }^{\text {a }}$ \\ ${ }^{a}$ Zhengzhou Institute of Surveying and Mapping, Zhongyuan District, Zhengzhou, 450000 Henan, China - \\ hou3938268@126.com,(gengxun_rs,xing972403)@163.com,xq@szdcec.com \\ ${ }^{\mathrm{b}}$ Information Science and Technology Institute, Jinshui District, Zhengzhou, 450000 Henan, China - tyh_983@126.com
}

Commission IV, WG IV/8

KEY WORDS: Mar Exploration, Radiometric Calibration, Field Program Gate Array (FPGA), Digital Signal Processor (DSP), Linear Pushbroom Imagery; HiRISE

\begin{abstract}
:
Due to the large data amount of HiRISE imagery, traditional radiometric calibration method is not able to meet the fast processing requirements. To solve this problem, a radiometric calibration system of HiRISE imagery based on field program gate array (FPGA) is designed. The montage gap between two channels caused by gray inconsistency is removed through histogram matching. The calibration system is composed of FPGA and DSP, which makes full use of the parallel processing ability of FPGA and fast computation as well as flexible control characteristic of DSP. Experimental results show that the designed system consumes less hardware resources and the real-time processing ability of radiometric calibration of HiRISE imagery is improved.
\end{abstract}

\section{INTRODUCION}

Mars is the closest planet to the earth in various characteristics and it is the planet that is the most likely to breed life within the solar system. Thus the mars exploration has become the focus for the deep space exploration in this century (Xu, 2006). At present, the countries that have successfully implemented Mars exploration are mainly European and American countries, but China, Japan, India and other countries also plan to carry out mars exploration. The Mars exploration tasks are divided into orbit detector and landing detector. The former can generate mars topographic data through the image data acquired during on-orbit service, such as American Viking, Mars Global Surveyor, Mars Reconnaissance Orbiter and European Mars Express (Albertz, 2005; Li, 2011 and Shan, 2005). The later acquires various samples through landing on the Mars for scientific research, such as American Spirit, Opportunity, Phoenix and Curiosity.

In various mars probes, the HiRISE camera (High Resolution Imaging Science Experiment) carried on Mars Reconnaissance Orbiter is the mars acquisition sensor with the highest image resolution (Kirk, 2008). This camera adopts multi-line splicing technology to extend the ground coverage and offers exquisite geographic data for site selection and mars scientific research. However, as affected by various factors during the imaging process, there are noises including dark current and flat field in the raw imagery of HiRISE. It seriously affects the image quality and it needs radiometric calibration. In addition, as for each linear CCD array of HiRISE, there are two channels to obtain the data. The gray level is inconsistent among channels. Thus the seamless splice shall be done for the CCD of each linear CCD array and then the complete imagery data can be acquired. At present, the HiRISE image radiometric calibration of NASA adopts the post processing technique.

The radiometric calibration of satellite imagery can be treated in real time. Giving consideration that the HiRISE image data volume is large, the power consumption is large in case of adopting general-purpose computer and it is difficult to meet the on-orbit real time processing demand. FPGA (Field Programmable Gate Array) has many advantages such as small size, low power consumption and strong parallel processing capability. Thus, for the purpose of realizing the on-orbit real time radiometric calibration for HiRISE image, the radiometric calibration of high resolution imagery based on FPGA is designed in this paper. For on-orbit radiometric calibration, we adopt a hardware structure of FPGA and DSP, and it fully makes use of the parallel processing capability of FPGA and the rapid calculation and flexible scheduling performance of DSP.

\section{RADIOMETRIC CALIBRATION OF HIRISE IMAGERY}

\subsection{Operating Principles for HiRISE Linear Camera}

The orbit altitude of the HiRISE camera carried on MRO is about $300 \mathrm{~km}$, the equivalent camera focal length is about $12 \mathrm{~m}$, and the ground sampling distance of the imagery is about $30 \mathrm{~cm}$. It can acquire blue-green, red and near-infrared band imagery and make use of these three band images to integrate the false color image for the surface of the Mars. To extend the ground coverage area, the camera adopts multi-linear array spicing technology, as shown in figure 1 . The red band image uses 10 linear CCD arrays, the blue-green and near-infrared band image use 2 linear CCD arrays separately, the number of pixels for each channel is 1024 and the complete CCD image will be formed after the spicing later.

\footnotetext{
* Corresponding author
} 


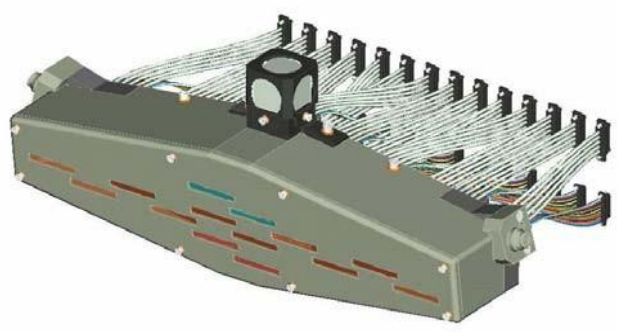

Figure 1. Mars HiRISE camera focal plane diagram (picture source: NASA official website)

To guarantee that the high quality and resolution imagery can be obtained under high speed flight, HiRISE camera adopts TDI (time delay integration) technology and the highest integration level is 128 . Within 1 second, it can obtain 13,000 scan lines, that is 76 microsecond for one scanning line. The quantification bit number of the imagery is 14 originally. As the storage resource is limited, in order to improve the data storage capacity, the LUT polling list can be used to compress the 14-digit data into 8-digit data.

\subsection{Radiometric Calibration Algorithm of HiRISE Imagery}

The HiRISE camera offers unprecedented high-resolution imagery for the research of Mars. However, as it is affected by various factors during the imaging process, there are dark current, flat field and other noises in the original imagery of HiRISE which seriously affects the quality of the imagery. Therefore, the radiometric calibration must be conducted. The radiometric calibration parameters of HiRISE imagery are shown in Table 1. These parameters are extracted by ISIS software (USGS, 2015).

\begin{tabular}{|c|c|c|}
\hline $\begin{array}{c}\text { Parameter } \\
\text { Description }\end{array}$ & $\begin{array}{c}\text { Parameter } \\
\text { Abbrevation }\end{array}$ & Parameters \\
\hline \multirow{2}{*}{$\begin{array}{c}\text { Dark field calibration } \\
\text { parameters }\end{array}$} & ZBF & ZeroBufferFit \\
\cline { 2 - 3 } & ZD & ZeroReverse \\
\hline Linear drifting gain & GLD & ZeroDark \\
\hline $\begin{array}{c}\text { Channel } \\
\text { normalization gain }\end{array}$ & GCN & GainChannelNormalize \\
\hline Non-linear gain & GNL & GainNonLinearity \\
\hline Flat field gain & GFF & GainFlatField \\
\hline Temperature gain & GT & GainTemperature \\
\hline Unit conversion gain & GUC & GainUnitConversion \\
\hline
\end{tabular}

Table 1. Parameters of radiometric calibration for HiRISE

As the original imagery data is compressed from 14 digits into 8 digits, the uncompressing processing shall be done before the radiometric calibration. For the convenience of calculation, we transfer the uncompressed data into 16 digits. Set the gray value of pixel of the original uncalibrated imagery as $i D N$, the gray value after the calibration as $o D N$, then the radiometric calibration formula of the HiRISE imagery is as below:

$$
\begin{aligned}
o D N= & (i D N-Z B F-Z R-Z D) / G L D \times G C N \times G N L \times \\
& G F F \times G T / G U C
\end{aligned}
$$

As each linear CCD array of HiRISE is composed by two channels and the images of these two channels have splicing seams after the radiometric calibration. Thus the image data of the two channels must be processed to eliminate the splicing seam. In this paper, it adopts histogram matching to eliminate the splicing seam and the specific procedures are as follows:

(1) Separately calculate the gray level histogram $H_{1}(i)$ and $H_{2}(i)$ in the two channels, then calculate the accumulated gray level histogram $H S_{1}(i)$ and $H S_{2}(i)$, of which $0 \leq 2^{16}-1$, $H S_{1}(i)=\sum_{j=0}^{i} H_{1}(j), H S_{2}(i)=\sum_{j=0}^{i} H_{2}(j)$.

(2) Based on the histogram $H_{1}(i)$ of one channel and the principle of taking the minimum difference of the accumulated gray level histogram, the gray level mapping rule of the imagery in the two channels is established to realize the matching of the image gray level histogram for the two channels.

(3) According to the mapping rule of the gray level among the imagery in the two channels, integrate the two channel images and construct a seamless linear array CCD imagery.

In conclusion, the radiometric calibration algorithm of HiRISE imagery is shown in figure 2 and the specific procedures are as follows:

(1) Preprocess HiRISE imagery, decompress the 8 digit compressed imagery into 16 digits and obtain parameters required for radiometric calibration.

(2) Import the depressed imagery data and radiometric calibration parameters into FPGA hardware platform.

(3) For each linear array CCD imagery, the radiometric calibration is conducted using the formula 1. Each linear array is practically divided into two channels and there are 28 files.

(4) For two CCD channel imagery on one linear array, make histogram matching to eliminate the effect of splicing seam and splice to form a complete line CCD array imagery.

(5) Export the linear array CCD imagery after the radiometric calibration and form 14 spliced linear CCD array imagery files.

As the histogram matching needs to utilize the whole scene imagery data, the on-orbit radiometric calibration can obtain the complete imagery and traverse on-orbit the whole scene imagery data and realize the histogram image matching. It can complete the imagery radiometric calibration and linear array CCD splicing without transmitting the imagery data to the ground. However, it has higher requirements on the storage capacity of the satellite.

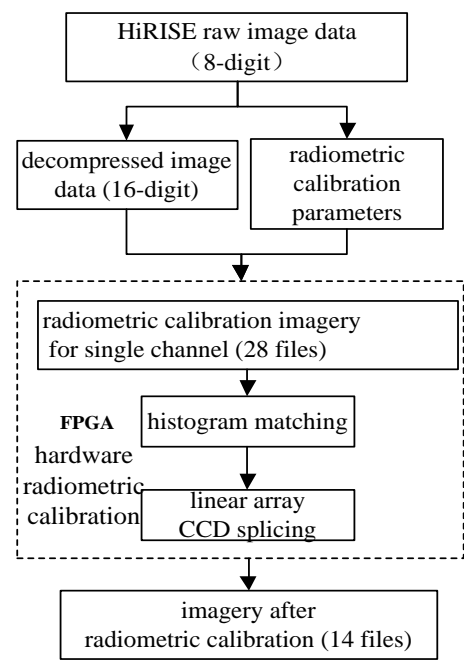

Figure 2. Radiometric calibration process for HiRISE imagery based on FPGA 


\section{RADIOMETRIC CALIBRATION SYSTEM DESIGN BASED ON FPGA}

The radiometric calibration shall be repeated for each pixel point and the data volume to be processed is very large. In consideration that FPGA is suitable for massive repeated and simple operations, FPGA can be adopted to realize the radiometric calibration and it can be done by the way of reading and calibration simultaneously. When the raw image data is read, the radiometric calibration is also processed. As the elimination of splicing seam needs to calculate the gray level histogram of the imagery data after the calibration, it needs to add 1 to the number of the pixel points with the such gray value till the completion of all pixel points. Thus the gray level histogram is also for making simple and repeated calculation and the FPGA also can be adopted. In consideration that the data bit width of the imagery after the calibration is 16 bit, namely, the number of the histogram statistic layers are 65,536. In addition, the data volume is large and it make the bit width of the storage required is comparatively big. Thus the storage space required for calculating the single channel imagery data histogram is relatively big and it adopts off-chip memory in design to store the statistical result of the gray level histogram. To store the histogram statistical result of the two channels, it needs two offchip memories. To obtain the mapping rule according to the histogram statistical result, it requires calling the histogram statistical result repeatedly and circularly and the control is relatively complex, thus it adopts DSP to calculate the histogram mapping rule, writes the result into another memorizer outside of FPGA and FPGA will write the processed image into the corresponding memorizer.

According to the aforesaid analysis, the HiRISE imagery calibration system designed is shown in figure 3 . The radiometric calibration module, histogram statistical module and gray level mapping module are realized by FPGA and the calculation of mapping rule is realized by DSP. Such division of the functional modules can make full use of the advantages of FPGA and DSP and improve the performance of the system.

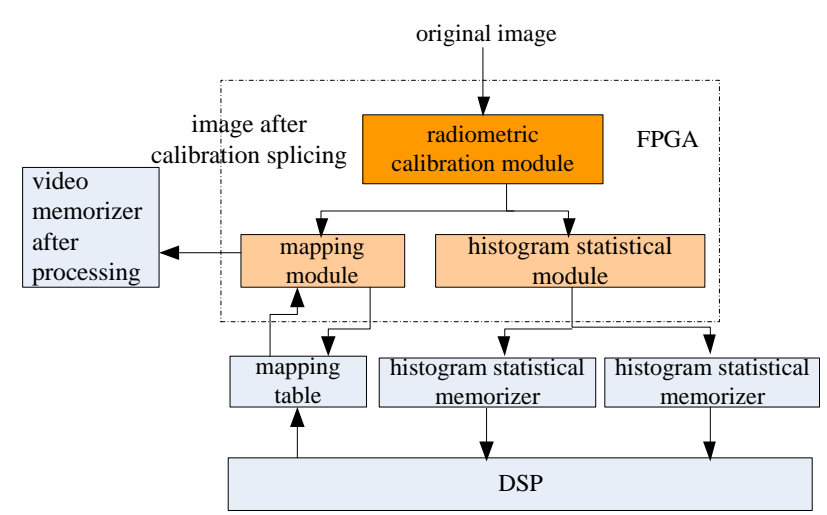

Figure 3. Structure diagram of calibration system for HiRISE imagery

\subsection{Radiometric Calibration Module}

We can see from the formula (1) that the raw image data of each pixel point needs two times of subtractions, two times of division operations and three times of multiplications to get the calibrated value. In addition, as the image data volume needed to be processed is large, the calculation amount is large. As the parameters other than $i D N$ in formula (1) are fixed values, then the formula (1) can be simplified into the form of formula (2):

$$
o D N=(i D N-\alpha) \times \beta
$$

Where

$$
\begin{aligned}
& \alpha=Z B F+Z R+Z D \\
& \quad \beta=G C M \times G N L \times G F F \times G T /(G L D \times G U C)
\end{aligned}
$$

We can see from the formula (2) that, the radiometric calibration for each pixel point needs one time of additive operation and one time of multiplication and thus it can adopt the hardware structure shown in figure 4 for radiometric calibration.

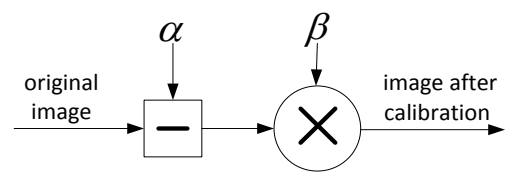

Figure 4. Radiometric calibration modular structure diagram

\subsection{Gray Level Histogram Statistical Module}

The gray level histogram is to calculate the number of the pixel points in each gray level within the whole imagery. However, the gray level is subject to the bit width of the pixel value. Set the gray value of the pixel point $(x, y)$ as $I(x, y)$ and the gray level as $H_{I}$. The subscript of $H_{I}$ corresponds to the gray value of the pixel point. We can adopt the structure diagram shown in figure 5 to realize the gray level histogram statistical module. Before the statistics, reset the histogram statistical memorizer, then when reading the gray value of one pixel point, take the pixel value as the address to read the din for corresponding position of the histogram statistical memorizer, add 1 to din and get the dout and then write the dout to the corresponding storage space of the address pixel value. Repeat the aforesaid operations until reading all the imagery data, and at that time the data stored in the histogram statistical memorizer is the statistical result of the gray level histogram.

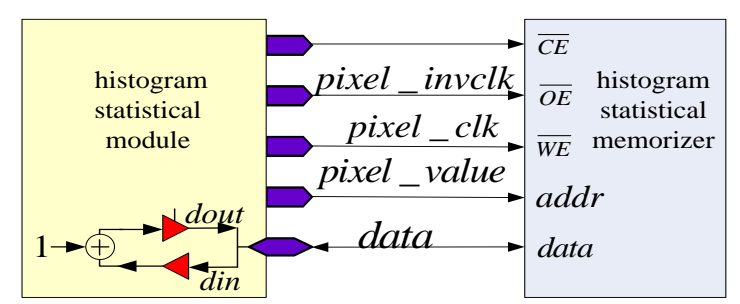

Figure 5. Statistical module diagram of gray level histogram

\subsection{Mapping Module}

In the mapping module, it inputs the image as per the mapping table calibration, namely, consider the gray value of the image input as the lookup address for the mapping table, take the value in the lookup table as the gray value of the image after the calibration, store it in the external storage space, and then complete the seamless splicing of the two channel images. Thus, the structure as shown in Fig. 6 can be adopted to realize the mapping module. DSP can obtain the mapping table for the two channel images through matching the gray statistical histogram of the two channel images, store it in the memorizer of the offchip mapping table and the FPGA can realize the seamless splicing for the two channel images through lookup the mapping table. DSP and FPGA share the data and control port of the mapping table memorizer and can realize the time sharing 
multiplex for DSP and FPGA by controlling the chip select signal.

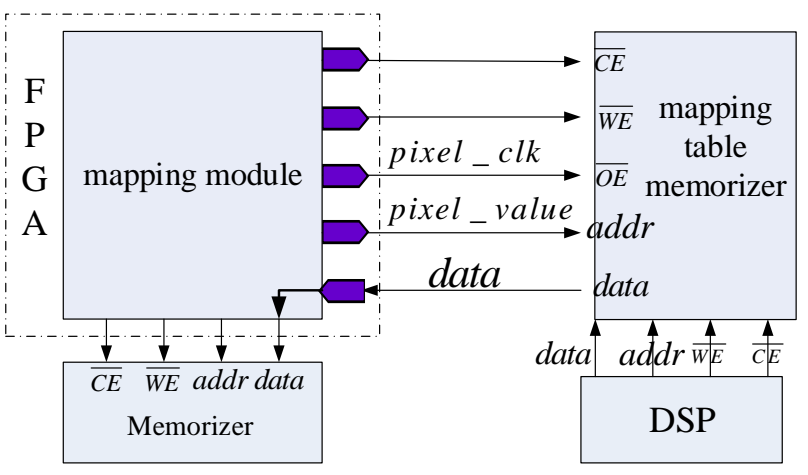

Figure 6. Mapping modular structural diagram

\section{EXPERIMENT AND ANALYSIS}

To verify the performance of the radiometric calibration system designed in this paper, the simulation experimental test is made. The experiment image was PSP_001521_2025 which was obtained on Nov. 22, 2006. It is located in the landing area of the lander of Viking1 and the resolution is about $30 \mathrm{~cm}$. After the image data is decompressed into 16 digits by ISIS software, the data volume in single channel is about $135 \mathrm{MB}$.

The mapping radiometric calibration designed in this paper needs 1 piece of FPGA, 1 piece of DSP and 3 pieces of memorizer and a comparatively large memorizer. The hardware resource consumed by the three functional modules realized by FPGA and the real-time performance are as shown in table 2 and table 3 . We can see from table 2 and table 3 that FPGA resources consumed by this system are few, but the maximum operating frequency is very high, which exceeds $200 \mathrm{Mhz}$. The data processing time of the radiometric calibration system based on FPGA is 4.36s. For comparison, the radiometric calibration processing of the same data by ISIS using computer is conducted and the processing time is $7.80 \mathrm{~s}$. The computer configuration is $8 \mathrm{~GB}$ of memory and Intel Core i5 CPU.

\begin{tabular}{|c|c|}
\hline Resource consumption & Value \\
\hline Qty of slice registers & 87 \\
\hline Slice lookup table & 104 \\
\hline
\end{tabular}

Table 2. Resource consumption table

\begin{tabular}{|c|c|}
\hline $\begin{array}{c}\text { Real-Time performance } \\
\text { comparison }\end{array}$ & Value \\
\hline Min. clock period & 4.36 \\
\hline Max. operating frequency & 229.373 \\
\hline
\end{tabular}

Table 3. Real time performance comparison table

The figure 7 illustrates the radiometric calibration result of HiRISE imagery. We can see that it eliminates the obvious strip noise in the imagery after the radiometric calibration and improves the quality of the imagery. The figure 8 is the result of elimination of splicing seam. Through the experiment, the feasibility of the radiometric calibration of the HiRISE imagery is verified.

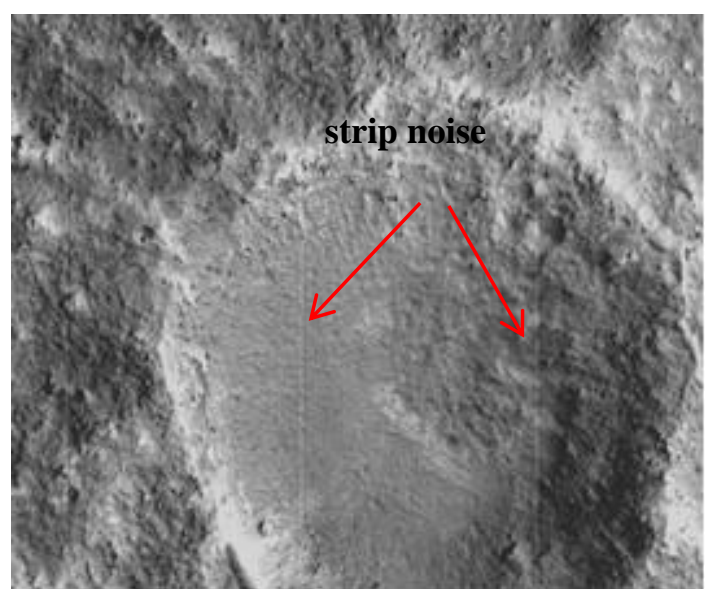

(a) image before radiometric calibration (1:1)

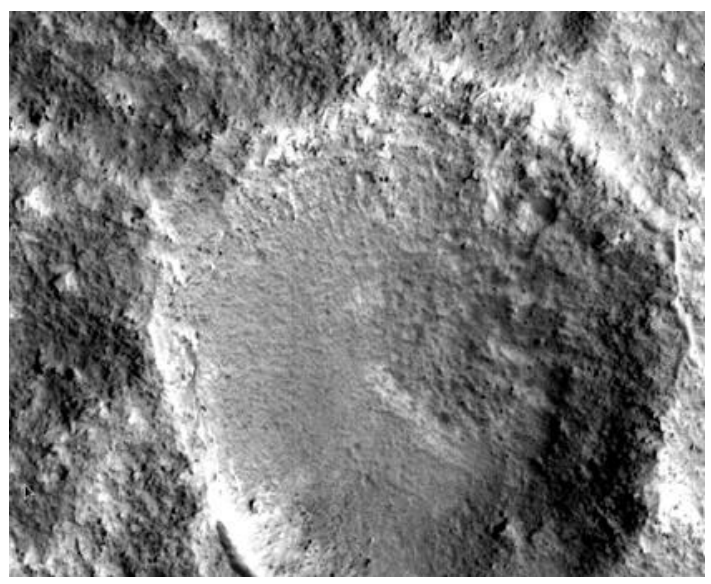

(b) image after radiometric calibration (1:1)

Figure 7. Radiometric calibration result of HiRISE imagery

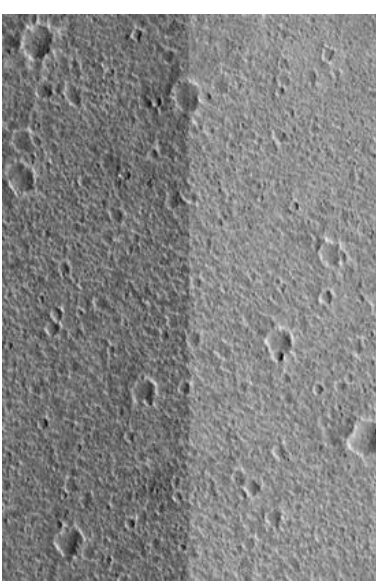

(a) before elimination

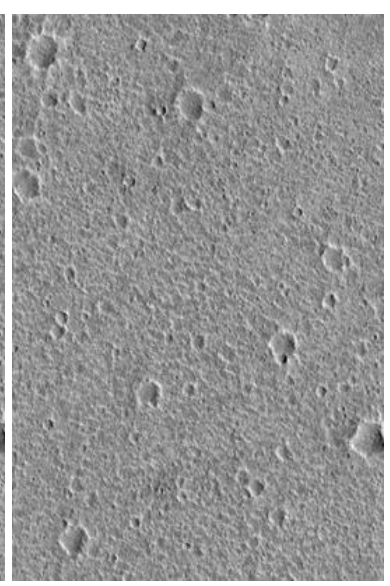

(b) after elimination

Figure 8. Elimination results of splicing seam

\section{CONCLUSIONS}

The radiometric calibration of HiRISE high resolution imagery is the basis for the subsequent data processing and analysis. As HiRISE adopts 14 linear CCD arrays for the splicing and each linear CCD array has multiple data channels, the radiometric calibration of HiRISE imagery is more difficult than other satellite imagery. To meet the on-orbit real time radiometric calibration demand for HiRISE imagery, we design a kind of radiometric calibration system on the basis of FPGA. This system 
adopts FPGA and DSP to coprocessing image data so as to improve the processing ability. The experimental result demonstrates that the on-orbit radiometric calibration system designed in this paper is characterized by less resource consumption and fast processing speed.

\section{ACKNOWLEDGEMENTS}

This work was supported by the National Natural Science Foundation of China (41371436, 41401533) and National Program on Key Basic Research Project of China (2012CB720000).

\section{REFERENCES}

Albertz, J., Attwenger, M., and Barrett, J., 2005. HRSC on Mars Express-photogrammetric and cartographic research. Photogrammetric Engineering and Remote Sensing, 71(10), pp. 1153-1166.

Kirk, R., L., Kraus, E., H., Rosiek, M., R., 2008. Ultra high resolution topographic mapping of Mars with MRO HiRISE stereo images: meter-scale slopes of candidate Phoenix landing sites. Journal of Geophysical Research, 113(3), pp.1-31.

Li., R., X., Hwangbo, J., Chen Y., H., and Di, K., C., 2011. Rigorous photogrammetric processing of HiRISE stereo imagery for Mars topographic mapping. IEEE Transactions on Geoscience and Remote Sensing, 49(7), pp.2558-2572.

Shan, J, Yoon, J., and Lee, D., S., 2005. Photogrammetric analysis of the Mars Global Surveyor mapping data. Photogrammetric Engineering and Remote Sensing, 71(1), pp. 97-108.

Xu, Q., 2006. Digital space and spacial survey and its supporting techniques. Journal of Zhengzhou Institute of Surveying and Mapping, 23(2), pp. 97-100.

USGS, 2015. USGS Integrated Software for Imagers and Spectrometers, America. http://isis.astrogeology.usgs.gov/ (10 Nov. 2015) 